\title{
Is immunotherapy the holy grail for pancreatic cancer?
}

\author{
Roland Andersson ${ }^{1}$, Carlos-Filipe Pereira ${ }^{2,3}$, Monika Bauden ${ }^{1}$ \& Daniel Ansari*,1 \\ ${ }^{1}$ Department of Surgery, Clinical Sciences Lund, Lund University \& Skåne University Hospital, Lund, Sweden \\ ${ }^{2}$ Molecular Medicine \& Gene Therapy, Lund Stem Cell Center, Lund University, BMC A12, 221 84, Lund, Sweden \\ ${ }^{3}$ Wallenberg Center for Molecular Medicine, Lund University, Lund, Sweden \\ *Author for correspondence: Tel.: +46 4622246 74; daniel.ansari@med.lu.se
}

\section{"One solution to improve the effectiveness of immunotherapy may rely on enhancing the immunogenicity of pancreatic tumor cells."}

First draft submitted: 16 September 2019; Accepted for publication: 30 October 2019; Published online: 21 November 2019

Keywords: biomarkers $\bullet$ immune checkpoint inhibitor $\bullet$ immunotherapy $\bullet$ pancreatic cancer $\bullet$ tumor antigenicity

Novel approaches to trigger the immune system against cancer have recently gained much attention. The pioneers within the field, James Allison (MD Anderson Cancer Center, USA) and Tasuku Honjo (Kyoto University, Japan), were awarded the Nobel Prize in Physiology or Medicine 2018 for their breakthrough research on CTLA-4 and PD-1/PD-L1, respectively. These contributions have been fundamental for the development of immune checkpoint blockade drugs and have transformed the treatment of patients with advanced melanoma and several other tumors [1-3]. Given the success of immunotherapy in several solid tumors, the question remains whether immunotherapy is also an option in a recalcitrant tumor such as pancreatic cancer?

\section{Immunotherapy \& pancreatic cancer}

Pancreatic cancer is currently the third leading cause of cancer death, and is projected to be the second cause of death in cancer within the next few years [4]. The lack of improvements in pancreatic cancer treatment is best illustrated by 50 years of stagnated survival rates in the Nordic countries [5]. This probably can be extrapolated to all other countries in the western world.

Immunotherapies are in clinical trials for pancreatic cancer individually or in combination. However, the success of these approaches is still limited in pancreatic cancer. Checkpoint inhibitors targeting CTLA-4 and PD-1/PD-L1 were inefficient as monotherapy in unselected patients with pancreatic cancer [6,7]. Only $1 \%$ of pancreatic tumors displaying microsatellite instability or mismatch repair deficiency seem to benefit from treatment $[8,9]$. Other immunotherapies, such as dendritic cell-based vaccines [10], chimeric antigen receptor T cells [11] and oncolytic viruses [12,13] have also been evaluated, but with limited success.

There are several potential mechanisms contributing to the lack of benefit of immunotherapy in pancreatic cancer. One important factor is the poor tumor antigenicity. This is partly related to the low mutation rate of pancreatic cancer with an average of 45 somatic mutations [14], which affects the production of neoantigens and immune recognition. In contrast, melanoma harbors approximately 135 somatic mutations. Neoantigen burden and quality are major determinants of tumor immunogenicity [15,16] and may explain the success of checkpoint inhibitors in melanoma. Another unique characteristic of pancreatic cancer is the desmoplastic, immunosuppressive and hypoxic microenvironment. The pancreatic tumor microenvironment comprises cancer-associated fibroblasts, endothelial cells, extracellular matrix proteins and immunosuppressive cells including myeloid-derived suppressor cells, Tregs and tumor-associated macrophages, lacking effector T cells.

\section{Future perspective for immunotherapy \& pancreatic cancer}

One solution to improve the effectiveness of immunotherapy may rely on enhancing the immunogenicity of pancreatic tumor cells. Downregulation of the antigen presentation machinery has been established as an important mechanism of immune evasion and is observed in pancreatic cancer [17]. Epigenetic mechanisms appear to underlie

Future $\because$ Medicine 
MHC-I downregulation impairing the ability of tumor cells to generate peptides from tumor antigens that can be processed and presented to cognate T cells [18]. Restoring or interfering with the MHC-I presentation pathway in pancreatic tumor cells may therefore open opportunities to increase antigenicity and responsiveness to immune checkpoint blockade [19,20]. Furthermore, immunotherapy may need to be combined with drugs that sensitize the tumor microenvironment by reprogramming the tumor vasculature and the immune compartment. These include myeloid-derived suppressor cells and Tregs, in order to overcome microenvironment-induced treatment resistance and enhance antitumor immune activity [21].

Importantly, there is an unmet need for developing predictive biomarkers of immunotherapy response to enable precision therapy. The recent molecular classification of pancreatic cancer indicates the possibility to use immunotherapy in subgroups of patients, at least from a theoretical point of view. An immunogenic subtype, involving upregulation of immune networks in acquired immune suppression, has been described [22]. Further characterization of the immunogenic subtype of pancreatic cancer has revealed subsets of $\mathrm{PD}-\mathrm{L} 1^{+} / \mathrm{CD} 8^{\text {high }}$ patients in whom inhibiting the PD-1 pathway may bolster CD8 ${ }^{+}$T-cell responses and lead to improved survival [23]. Additional predictive variables may need to be evaluated in combination in order to generate individual immune profiles. These factors include tumor mutation burden, copy number variation, human leukocyte antigen (HLA) class diversity, T-cell repertoire, TGF- $\beta$ expression and tumor microbiome [24,25].

The future of cancer immunotherapy may take benefit from innovations in computational models and biotechnology. One option is to integrate molecular datasets and machine-learning algorithms. For example, machine learning was applied to molecular data generated from massively parallel sequencing of mutations within melanoma tumors in order to predict those mutated peptides that bind with high affinity to HLA molecules [26]. Using this approach, immunogenic vaccines could be generated, which target the predicted neoantigens. The results showed that four of six vaccinated melanoma patients had no recurrence 2 years after vaccination, while two patients with progressive disease were treated with anti-PD-1 treatment and demonstrated complete tumor regression.

Another option is to make use of novel methods to optimize drug delivery and targeting. Many solid tumors are characterized by a 'leaky' vasculature and an abundance of extracellular matrix proteins, such as collagen. Immune checkpoint inhibitors conjugated to a collagen-binding domain and fused to IL-2 have been shown to effectively target the tumor, enhance treatment efficacy and improve immune activation within the tumor microenvironment [27]. Furthermore, nanotechnology can improve the spatio-temporal release of immunotherapy, by enhancing biodistribution and extending local drug release [28]. For example, nanoparticles have been generated that are MMP2 sensitive, carry a photosensitizer and are conjugated to PD-L1 antibodies [29]. These nanoparticles accumulate at the tumor site, which triggers release of the antibody. Near-infrared radiation activates the photosensitizer to produce reactive oxygen species, which sensitizes the cancer cells to PD-L1 blockade.

Finally, molecular imaging may become important in order to visualize immunotherapy targets within tissues, improve patient selection and monitor treatment. Immuno-PET is a noninvasive method that combines positron emission tomography with radiolabeled antibodies, proteins or peptides in order to map cell-surface molecular targets. Several clinical trials are underway to determine the benefit of immuno-PET to monitor responses to immunotherapy [30]. In the preclinical setting, novel imaging models have been developed using, for example, nanoparticles for visualization of multiple immune targets at the same time [31]. Additional studies are needed to translate these diagnostic approaches to the clinical setting.

\section{Conclusion}

In conclusion, immunotherapeutic intervention in pancreatic cancer has so far rendered little success. General limitations that may explain lack of efficacy includes poor immunogenicity, highly immunosuppressive microenvironment and lack of reliable stratifying biomarkers. An increasing number of clinically available immunotherapeutic agents are opening new avenues to harness the immune system against pancreatic tumors. Improvement might be obtained by approaches to reprogram tumor cells and their microenvironment for immunotherapy, as well as development of predictive biomarker models and molecular imaging, in order to enable precision therapy and overcome drug resistance.

Financial \& competing interests disclosure

The authors have no relevant affiliations or financial involvement with any organization or entity with a financial interest in or financial conflict with the subject matter or materials discussed in the manuscript. This includes employment, consultancies, honoraria, stock ownership or options, expert testimony, grants or patents received or pending, or royalties.

No writing assistance was utilized in the production of this manuscript. 


\section{Ethical conduct of research}

The authors state that they have obtained appropriate institutional review board approval or have followed the principles outlined in the Declaration of Helsinki for all human or animal experimental investigations.

\section{References}

1. Hodi FS, Chiarion-Sileni V, Gonzalez R et al. Nivolumab plus ipilimumab or nivolumab alone versus ipilimumab alone in advanced melanoma (CheckMate 067): 4-year outcomes of a multicentre, randomised, Phase III trial. Lancet. Oncol. 19(11), 1480-1492 (2018).

2. Hellmann MD, Ciuleanu TE, Pluzanski A et al. Nivolumab plus ipilimumab in lung cancer with a high tumor mutational burden. $N$. Engl. J. Med. 378(22), 2093-2104 (2018).

3. Rini BI, Plimack ER, Stus V et al. Pembrolizumab plus axitinib versus sunitinib for advanced renal-cell carcinoma. N. Engl. J. Med. 380(12), 1116-1127 (2019).

4. Rahib L, Smith BD, Aizenberg R, Rosenzweig AB, Fleshman JM, Matrisian LM. Projecting cancer incidence and deaths to 2030: the unexpected burden of thyroid, liver, and pancreas cancers in the United States. Cancer Res. 74(11), 2913-2921 (2014).

5. Ansari D, Tingstedt B, Andersson B et al. Pancreatic cancer: yesterday, today and tomorrow. Future Oncol. 12(16), 1929-1946 (2016).

6. Johansson H, Andersson R, Bauden M, Hammes S, Holdenrieder S, Ansari D. Immune checkpoint therapy for pancreatic cancer. World J. Gastroenterol. 22(43), 9457-9476 (2016).

7. Hilmi M, Bartholin L, Neuzillet C. Immune therapies in pancreatic ductal adenocarcinoma: where are we now? World J. Gastroenterol. 24(20), 2137-2151 (2018).

8. Humphris JL, Patch AM, Nones K et al. Hypermutation in pancreatic cancer. Gastroenterology 152(1), 68-74.e62 (2017).

9. Luchini C, Bibeau F, Ligtenberg MJL et al. ESMO recommendations on microsatellite instability testing for immunotherapy in cancer, and its relationship with PD-1/PD-L1 expression and tumour mutational burden: a systematic review-based approach. Ann. Oncol. 30(8), 1232-1243 (2019).

10. Deicher A, Andersson R, Tingstedt B, Lindell G, Bauden M, Ansari D. Targeting dendritic cells in pancreatic ductal adenocarcinoma. Cancer Cell. Int. 18, 85 (2018).

11. Hou B, Tang Y, Li W, Zeng Q, Chang D. Efficiency of CAR-T therapy for treatment of solid tumor in clinical trials: a meta-analysis. Dis. Markers 2019, 11 (2019).

12. Hecht JR, Bedford R, Abbruzzese JL et al. A Phase I/II trial of intratumoral endoscopic ultrasound injection of ONYX-015 with intravenous gemcitabine in unresectable pancreatic carcinoma. Clin. Cancer Res. 9(2), 555-561 (2003).

13. Mulvihill S, Warren R, Venook A et al. Safety and feasibility of injection with an E1B-55 kDa gene-deleted, replication-selective adenovirus (ONYX-015) into primary carcinomas of the pancreas: a Phase I trial. Gene Ther. 8(4), 308-315 (2001).

14. Vogelstein B, Papadopoulos N, Velculescu VE, Zhou S, Diaz LA Jr, Kinzler KW. Cancer genome landscapes. Science 339(6127), 1546-1558 (2013).

15. Balachandran VP, Luksza M, Zhao JN et al. Identification of unique neoantigen qualities in long-term survivors of pancreatic cancer. Nature 551(7681), 512-516 (2017).

16. Van Allen EM, Miao D, Schilling B et al. Genomic correlates of response to CTLA-4 blockade in metastatic melanoma. Science 350(6257), 207-211 (2015).

17. Pandha H, Rigg A, John J, Lemoine N. Loss of expression of antigen-presenting molecules in human pancreatic cancer and pancreatic cancer cell lines. Clin. Exp. Immunol. 148(1), 127-135 (2007).

18. Cai L, Michelakos T, Yamada T et al. Defective HLA class I antigen processing machinery in cancer. Cancer Immunol. Immunother. 67(6), 999-1009 (2018).

19. Garrido F, Aptsiauri N, Doorduijn EM, Garcia Lora AM, Van Hall T. The urgent need to recover MHC class I in cancers for effective immunotherapy. Curr. Opin. Immunol 39, 44-51 (2016).

20. Rosa FF, Pires CF, Kurochkin I et al. Direct reprogramming of fibroblasts into antigen-presenting dendritic cells. Sci. Immunol. 3(30), eaau4292 (2018).

21. Datta M, Coussens LM, Nishikawa H, Hodi FS, Jain RK. Reprogramming the tumor microenvironment to improve immunotherapy: emerging strategies and combination therapies. Am. Soc. Clin. Oncol. Educ. Book 39, 165-174 (2019).

22. Bailey P, Chang DK, Nones K et al. Genomic analyses identify molecular subtypes of pancreatic cancer. Nature 531(7592), 47-52 (2016).

23. Danilova L, Ho WJ, Zhu Q et al. Programmed cell death ligand-1 (PD-L1) and CD8 expression profiling identify an immunologic subtype of pancreatic ductal adenocarcinomas with favorable survival. Cancer Immunol. Res. 7(6), 886-895 (2019).

24. Havel JJ, Chowell D, Chan TA. The evolving landscape of biomarkers for checkpoint inhibitor immunotherapy. Nat. Rev. Cancer. 19(3), 133-150 (2019).

25. Riquelme E, Zhang Y, Zhang L et al. Tumor microbiome diversity and composition influence pancreatic cancer outcomes. Cell 178(4), 795-806.e712 (2019). 
26. Ott PA, Hu Z, Keskin DB et al. An immunogenic personal neoantigen vaccine for patients with melanoma. Nature 547(7662), 217-221 (2017).

27. Ishihara J, Ishihara A, Sasaki K et al. Targeted antibody and cytokine cancer immunotherapies through collagen affinity. Sci. Transl. Med. 11(487), eaau3259 (2019).

28. Goldberg MS. Improving cancer immunotherapy through nanotechnology. Nat. Rev. Cancer 19(10), 587-602 (2019).

29. Wang D, Wang T, Yu H et al. Engineering nanoparticles to locally activate T cells in the tumor microenvironment. Sci. Immunol. 4(37), eaau6584 (2019).

30. Marciscano AE, Thorek DLJ. Role of noninvasive molecular imaging in determining response. Adv. Radiat. Oncol. 3(4), 534-547 (2018).

31. Zhong $\mathrm{Y}, \mathrm{Ma} \mathrm{Z}$, Wang $\mathrm{F}$ et al. In vivo molecular imaging for immunotherapy using ultra-bright near-infrared-IIb rare-earth nanoparticles. Nat. Biotechnol. doi:10.1038/s41587-019-0262-4 (2019) (Epub ahead of print). 